\title{
LOS ASHAB AL-SIKKA DE 'ABD AL-RAHMĀN III, SEGUN IBN HAYYAN Y EL TESTIMONIO DE LAS MONEDAS
}

\author{
Alberto Canto García
}

La presencia de nombres propios en la moneda andalusí, es un hecho que se produce ya en la moneda del emirato omeya, por lo menos y con regularidad desde el siglo III H./ IX d. C., aunque existe alguna presencia anterior. Sin embargo se desconoce la función o el nivel administrativo que desempeñan dichas personas en el emirato, mientras que sobre los nombres que aparecen en la moneda califal poseemos algo más de información.

Como en tantas ocasiones fue Codera quien, por vez primera, analizó de manera cuidadosa estos nombres, en un trabajo de importancia capital (1). Su estudio le permitió confeccionar una lista de los nombres de personajes que aparecían en la moneda andalusí de los siglos X-XI d. C., y en el caso concreto del período de 'Abd al-Raḥmān III, constar que cuatro nombres aparecían citados como el șāhib al-sikka, es decir era los "prefectos de la ceca" de Córdoba y posteriormente de Madīna al-Zahrā' (2). Esta confirmación, por las fuentes, del cargo que correspondía a estos nombres daba pie a sospechar que todos los demás nombres, además de ellos, podían desempeñar esa misma función. Sin embargo ante la ausencia de una fuente que nos proporcionara la lista completa de los așhab al-Sikka, la relación establecida en época de Codera se articula combinando las monedas con el texto de Ibn 'Iḍārī.

A. Vives y G. Miles también dedicaron una parte de su trabajo a estudiar estos nombres, ya fuera completando la lista o buscando su identificación. El primero de ellos añade nombres a la lista conformada por Codera, gracias a piezas de su colección (3), mientras que el segundo sintetiza el estado de la cuestión e incide en la identificación de los personajes (4).

(1) Codera, F.: Titulos y nombres propios en las monedas arábigo-españolas, (1878), Madrid. En su trabajo Codera se basa sobre todo en el texto de Ibn 'Id̄ārī, gracias al cual identificó a Yaḥyà b. Yūnus, Aḥmad b. Muḥammad, 'Abd Allāh y 'Abd al-Rahmān b. Yahyà. Cfr. Ibn 'Idārī, Al Bayan al-Mugrib, I, II, éd. Dozy, R., (1848-1851), Leyden, Trał. E. Fagnan (1901-4), Argel, págs. 327.

(2) Codera, F.: Tratado de Numismática arábigo-española, (1879), Madrid, págs. 80, 84 y en especial 85; del mismo Titulos..., págs. 50-55 y 57.

(3) Vives, A.: Monedas de las dinastías arábigo-españolas, (1893), Madrid, págs. XIV-XVII, donde aparece 'Abd Allah en el 329 y Sa'id en el $330 \mathrm{H}$.

(4) Miles, G. C.: The coinage of the Umayyads of Spain, (1950), New York, págs. 55 y ss. 
De lo que no cabe duda, es que la presencia sistemática de estos nombres constituye una característica de la política monetaria de 'Abd al-Rahmān III, quizás como un producto de su reorganización del Estado y de la reapertura de la ceca de Córdoba en el año 316 H. (5). Los nuevos datos aportados en el Muqtabis V de Ibn Hayyan son de especial interés en este tema, como puede apreciarse en el siguiente fragmento, relacionado con la ceca y sus "prefectos":

«... La primera designación en la ceca recayó en Aḥmad b. Muhammad b. Musa b. Hudayr, luego en Yahyyà b. Yūnus al-Qabrī en el 320 (932), luego Muḥammad b. Futays, en el 321 (933), luego Sa'îd b. Yassās, en el 322 (934), luego su hermano 'Abd Allāh en el 327 (938-939), y luego de nuevo Sa'îd, quien traicionó su obligación y cometió fraude, que fue descubierto al investigar sus acuñaciones, mereciendo el enojo y la cárcel. Luego se ocupó de la ceca Qāsim b. Jālid, autor de la excelente acuñación que es hasta hoy proverbial, muerto luego por mano de sus esclavos en du-l-qada del año 332 (25 junio - 24 julio 944). An-Nasir nombró en su lugar a su primo 'Abd al-Rahmān b. Yahyà el sordo, luego a Muhammad b. Ahmad b. Hudayr, y luego a 'Abdallāh b. Muhammad al-Jarrūbī.

Posteriormente, an-Nasir trasladó la ceca a az-Zahrā, su nueva fundación, al ir a vivir allí, dejando de usar la de Córdoba y cerrando sus puertas, al sustituirla por la de az-Zahra' y llevarse allí la ceca, que confió a 'Abdarrahmān b. Yahyà, y allí continuó la acuñación...».

Por primera vez tenemos una relación casi completa de los ashab al-sikka del reinado de 'Abd al-Raḥmān III, incluso con los años (en algunos casos) en que desempeñan el puesto, la cual podemos comparar con el testimonio de las monedas; para ello vamos a seguir la lista cronológica que aporta Ibn Hayyān, mencionando las anomalías que aparezcan. Existen algunas diferencias, pues Ibn 'Iḍārī menciona cuatro prefectos (Yahyà $b$. Yūnus sustituyendo a Aḥmad b. Muḥammad en el 320, y 'Abn Allāh b. Muḥammad lo es por 'Abd al-Raḥmān b. Yaḩyà b. Idrīs en el 336), de los que conocemos monedas, con su nombre, del primer y tercero de los mencionados, las que corresponden al período de Ahmad no llevan nombre de prefecto y del último de ellos no existe ninguna moneda con su nombre, y en el lugar reservado aparece otro nombre. Por su parte Ibn Hāyyàn, en la lista más completa que se nos ha conservado, nos proporciona nueve nombres aunque alguno de ellos no llegue a acuñar ninguna moneda (caso de 'Abd al-Raḥmān b. Yahyà en el año 332, y en su segundo período, del 336). Si acudimos, por fin, a las monedas mismas, vemos que éstas recogen once nombres, que en su mayoría coinciden con los de las fuentes, pero en otros no.

316-319 H. (928/9-931 d. C.). Aḥmad b. Muḥammad b. Hudayr, designado como primer șāhib al-Sikka de Córdoba, sin embargo, su nombre no figura en ninguna de las monedas que de dicho período conservamos, por lo que debemos considerar que en este período inicial de la moneda califal, la política monetaria (en este as-

(5) Crónica Anónima de Abd l-Rahman III al-Nasir, ed. y trad. por E. Levy Provencal y E. García Gómez (1950), Madrid-Granada, págs. 153-154, cfr. Ibn Hayyan, Crónica del Califa 'Abdarrahman III an-Nasir entre los años 912 y 942 (Al-Muqtabis V), trad. por M. Jesús Viguera y F. Corriente (1981), Zaragoza, págs. 185-186. 
pecto) no está muy definida (6). A pesar de ello los testimonios de las fuentes son muy claros al respecto, y no tenemos porqué dudar en este punto, de su ausencia en el campo de las monedas. Miles consideró que el nombre de Ahmad b. Muhammad no aparece porque dicho privilegio no alcanza todavía al șāḥib al-sikka (7).

320 H. (932 d. C.). Yahyà b. Yūnus al-Qabrī, primero de los ạshab al-sikka que coloca su nombre en las monedas, y además de forma extensa; identificado ya por Codera (8), es sin duda la misma persona que en el año 320, firma sólo con Yahyà. Miles corroboró la identificación de Codera, pero atribuía la serie de feluses con nombre Yahyà, al período 306-320 H., sin concretar. Es evidente que todos los datos confirman que las monedas con nombre Yahyà o Yahyà b. Yũnus, sólo pueden corresponder al año 320 , con lo que se confirma esta pequeña duda y se realiza una datación más exacta (9).

321 H. (933 d. C.) Muhammad b. Fuṭays, quien, para Ibn Hayyān, sólo acuña en el $321 \mathrm{H}$. De hecho la totalidad de las monedas que conocemos de este año llevan su nombre y no ha aparecido ninguna moneda con él, en años anteriores; la creencia de Miles sobre una moneda con este nombre en el año 320 debe ser un error (10).

322-330 H. (933/4-942 d. C.). Sa'īd b. Ŷassās (11); es uno de los personajes de los que el Muqtabis nos ha dado una gran información. Por un lado hay que ampliar el margen cronológico que le atribuía Codera, pues sus emisiones alcanzan hasta el año $330 \mathrm{H}$.; Miles ya lo hizo sosteniendo sólo una ligera duda sobre el ejemplar del año 330, de la Col. Vives (12). El párrafo de Ibn Hayyān es claro y la única duda estaba en aclarar si Sa'īd acuñó o no moneda en el año de su sustitución, duda aclarada pues el ejemplar del MAN, es muy claro, y además existe otro ejemplar semejante en una colección particular. La segunda cuestión que se plantea en este período es la existencia de una moneda con nombre 'Abd Allāh, cuya fecha es del año 329 H. (13). Como hemos visto Ibn Hayyān cita que Sa'îd fue sustituido por su hermano 'Abd Allāh b. Ŷassās, en el año 327; pero todas las monedas de dicho año llevan el nombre Sa'īid. Aparece pues una divergencia entre lo que expone la fuente y el testimonio de las monedas, que se reduce a la fecha en que se produce la sustitución temporal, que debió ser breve, de todas formas, pues tanto en el año 327 como en el 329 (las dos fechas posibles) aparecen monedas de Sa'id.

(6) No olvidemos que en este período de cuatro años se suceden diferentes modelos de monedas, en la búsqueda de uno de ellos que se afirme como el modelo califal, en contraposición del modelo emiral anterior, y que refleje la nueva situación que se está desarrollando en Córdoba.

(7) Miles, G. C.: The coinage..., pág. 86; La fundación de la ceca y el nombre del primer director de la ceca están recogidos en Crónica Anónima, págs. 153-4.

(8) Codera, F.: Tútulos..., pág. 51. La forma correcta del nombre parece Yãhyà b. Yūnus al-Qabrí.

(9) Miles, G. C.: The coinage..., págs. 86 y 248-9, n. ${ }^{\circ} 199 \mathrm{e}, \mathrm{f}$ y g; Vives, 370, 374 y 375.

(10) Miles, G. C.: The coinage..., pág. 76 y n. $195 \mathrm{j}$. La atribución de Miles es errónea, puesto que Codera, F.: Tratado..., pág. 302, en el Apéndice XIII, sitúa la moneda de la col. Camarino en el año 320, pero con nombre Yâhyà, y en la tabla de nombres de la página 85, tampoco asigna el nombre Muhammad al año 320.

(11) Agrupo todos los años de Sa'ıd en una misma secuencia, aunque según Ibn Hayyān, en el 327 se interrumpe en favor de su hermano 'Abd Alläh, para volver en el 328.

(12) Miles, G. C.: The coinage..., pág. 65 y n. 217c. Vives, A.: Monedas..., pág. XVI, n. 391.

(13) Vives, A.: Monedas..., n. ${ }^{\circ} 392$. Miles, G. C.: The coinage..., n. ${ }^{\circ} 215 \mathrm{c}$. 
A pesar de esta duda, el texto de Ibn Hayyān confirma la presencia de 'Abd Allāh b. Ŷassās en la ceca en el año 327 (que se ratifica con la moneda de la col. Vives, aunque la fecha sea objeto de discusión), al tiempo que nos aclara la identidad del personaje. Este último punto es interesante, pues Miles había considerado al 'Abd Allāh (b. Ŷassās) del 329 H., como la misma persona que en 335-336 H. firma las monedas con el mismo nombre 'Abd Allāh (en este caso b. Muhammad) (14).

330-332 H. (941/2-943/4 d. C.), Qasim b. Jālid, personaje célebre (por lo menos en las monedas) del que conocemos muchos más datos de los que dispusieron Codera o Miles (15). De todas formas ninguna de las atribuciones realizadas por Miles son acertadas (16).

332-334 H. (943/4-945/6). Ibn Hayyān nos menciona a 'Abd al-Rahmān b. Yạ̣yà el sordo (es la primera de las dos ocasiones en que el autor del Muqtabis le coloca al frente de la ceca), siendo sucedido en el puesto por Muhammad b. Ahmmad b. Hudayr. Del primero de ambos no conocemos monedas, por lo que debemos pensar que su permanencia en la ceca debió ser muy breve, máxime si comprobamos que en el mismo año 332 aparecen las primeras emisiones de Muhammad con su nombre inscrito en el lugar correspondiente. Como sabemos que Qāsim b. Jālid fue asesinado en ḍu l-qa'da del 332 (25 de junio - 24 de julio del 944), los dos nombramientos y las primeras acuñaciones de Muhammad se tuvieron que realizar en los meses restantes antes del año 333.

335-336 H. (946/7-947/8). El personaje que aparece en el Muqtabis es 'Abd b. Muhammad al-Jarrūbī, uno de los ashab al-sikka identificados por Ibn 'Idārī: su destitución y prisión anteceden al cierre de la ceca en Córdoba y su traslado a la ciudad palatina de al-Zahrä, en donde se reanudan las emisiones pero con el nombre del siguiente encargado de la ceca. La identidad que de él nos hace Ibn Hayyān permite diferenciarlo de 'Abd Allāh b. Ŷassās, como ya hemos visto.

336-346 H. (946/7-958/9 d. C.) Ibn Hayyān (al igual que Ibn 'Iḍārī) cita como sahib a 'Abd al-Rahmān b. Yahyà, casi con toda seguridad, el mismo personaje que aparece en el año 332 y que no llega a acuñar ninguna moneda. Se repite el fenómeno que ocurre en el año mencionado, pues todas las monedas del año 336 al 346 H., llevan el nombre Muhammad en el campo. Codera ya percibió esta anomalía y la única solución que vislumbró fue la de suponer un cambio en la dirección de la ceca que justificase la presencia del nombre Muhammad (17). No disponemos de ningún elemento que nos permita apoyar esta hipótesis, a no ser la simi-

(14) Miles, G. C.: The coinage..., pàg. 70.

(15) Sobre Qāsim b. Jālid puede verse Chalmeta, P.: «Precisions au sujet du monnayage hispano-árabe (dirham qāsimí et dirham arba'ini)" en JESHO XXIV (1981), part. III, págs. 316-324; Vallvé, J.: "Notas sobre metrología hispano-árabe III. PESOS Y MONEDAS» en AL-QANTARA V (1984), págs. 147-168 y Canto, A.: "La reforma monetaria de Qāsim" (en prensa en AL-QANTARA). En ellos puede verse la abundante bibliografía sobre este personaje, así como algunas de las cuestiones suscitadas durante el final de la etapa de $\mathrm{Sa}^{\top} \bar{i} \mathrm{~d}$.

(16) Miles, G. C.: The coinage..., págs. 75-76, aunque interpretó de manera correcta la utilización del término "qāsimi" con un sentido de denominación genérica.

(17) Codera, F.: Títulos..., págs. 56-57. 
litud que guarda con lo acaecido en el año 332: nombramiento de 'Abd al-Raḥmān b. Yahyà y su sustitución por otra persona como șāhib al-sikka, que es quien realmente firma las monedas. Aquí el Muqtabis no ofrece la misma solución, pero que se repita la misma combinación de nombres resulta sorprendente; ante la falta de información sobre este problema, el último eslabón que une a esta pareja de nombres es, la ya comentada, sucesión de cuatro años antes. Podría pensarse que ocurra un fenómeno similar y el Muhammad que figura del 336 al 346 sea Muhammad b. Ahmad b. Hudayr.

346-350 H. (958/9-961 d. C.). Los cuatro últimos años del reinado de 'Abd alRaḥmān III, contemplan en el cargo de șāhib al-sikka, a un personaje de nombre Ahmad. Se trata del único de la serie que no viene citado por Ibn Hayyãn, ni por otra fuente, siendo las monedas su único testimonio; quizás por ello sea del que menos sabemos, pues ni Codera ni Miles pudieron identificarle (18).

Mención aparte merecen las monedas que en los años 334 y 335 H. (945/6-946/7 d. C.) aparecen con el nombre de Hišām en el campo. Codera ya advirtió ciertas peculiaridades en esta serie, a la que considera una interrupción de la secuencia normal de los ashab al-sikka, que enlaza perfectamente. Le llamó, sobre todo, la atención la distinta posición en que aparece el nombre de este personaje, por lo que se inclinaba a creer que esta serie pudiera estar relacionada (o controlada) por una persona distinta de los habituales prefectos de la ceca (19). Hemos dejado para el final este grupo porque estamos de acuerdo con la opinión de Codera y consideramos que estas monedas constituyen una emisión especial realizada por algún motivo que desconocemos; por ello el nombre que figura no aparece en la relación de ashab al-sikka que nos proporciona Ibn Hayyān. Hemos podido comprobar como el Muqtabis proporciona una información fiable y muy ajustada con los datos que nos proporcionan las mismas monedas y el nombre de Hišām es el único que no figura en la mencionada fuente (20). Si aceptamos la posibilidad de que el nombre de la persona no sea el de uno de los aṣab al-sikka, entonces no existe ninguna razón para que estuviera presente en la lista que los recoge, y por otra parte, la sensación que produce dicha serie de monedas es la de ser una emisión paralela, que no altera las acuñaciones normales de la ceca de Córdoba (21).

Una vez realizada esta revisión de la lista de los ashab al-sikka, podemos apreciar la exactitud de los datos que nos proporciona Ibn Hayyān en su obra; con la

(18) Miles, G.: The coinage..., págs. 55-56. Codera, F.: Títulos..., pág. 51.

(19) Codera, F.: Títulos..., págs. 55 y 56. La suposición sobre un segundo hijo de an-Nāşir de nombre Hił̌ām (pues un primero con dicho nombre ya había fallecido años antes), que fuera quien figurase en las monedas, tiene muy poca consistencia; cfr. Miles, G.: The coinage..., págs. 82-83, para quien le resulta chocante que alguien ocupase el cargo sólo para un espacio de tiempo tan corto. Tampoco es un argumento consistente, pues ya hemos visto algún prefecto de ceca, que sólo permanece un año en el puesto.

(20) Tampoco lo hace el de Ahmad, como ya hemos indicado, pero en el Muqtabis se encuentran muy bien recogidos los años anteriores al traslado de la ceca a az-Zahrā', y resulta muy raro un silencio total sobre un sahib al-sikka que desempeña su misión justo en esos años.

(21) Un trabajo sobre las emisiones de Hisam de los años 334-335 H., lo hemos presentado en el X Cong. Internacional de Numismática celebrado en Londres, en septiembre de 1986. Cfr. Canto, A.: ¿Una emisión conmemorativa de 'Abd al-Raḥmān III an-Nāṣirir?, (en prensa). 
excepción del último nombre de la lista, aparecen citados, con sus nombres completos, nueve prefectos de la ceca, quienes son corroborados por las monedas en ocho ocasiones. El noveno que no aparece en ninguna moneda, se trata (como ya sabemos) de 'Abd al-Rahmān b. Yahyà, tanto para su período del 332, como en el del 336 H. (22).

Se han identificado, de manera correcta, personas cuyos nombres eran semejantes, al aparecer sólo el alam, en las monedas; tal es el caso de 'Abd Allāh b. Ŷassās (327 H.) y 'Abd Allāh b. Muḥammad al-Jarrūbī (335-336 H.), y el de Muhammad b. Fuțays (321 H.) con Muhammad b. Ahmad b. Hudayr (332-334 H.), con la posibilidad de que se trate de la misma persona que firma monedas para el período 336-346 $\mathrm{H}$.

Conocemos el nombre de algún șāhib al-sikka que no llega a acuñar, por permanecer poco tiempo en el cargo, como es el caso de 'Abd al-Rahmān b. Yahyà el sordo (23).

Por lo tanto y a la vista de lo expuesto, la lista de los ashab al-sikka de 'Abd al-Rahmān III, queda de la siguiente manera:

Ahmad b. Muhammad b. Hudayr del 316 al $319 \mathrm{H}$.

Yahyà b. Yúnus al-Qabrī en el $320 \mathrm{H}$.

Muhammad b. Fuțays para el $321 \mathrm{H}$.

Sa'ìd b. Ŷassās en los años 322 al $327 \mathrm{H}$.

'Abd Allāh b. Ŷassās en el 327.

Sa'ìd b. Ŷassās del 327 al $330 \mathrm{H}$.

Qasim b. Jalid del 330 al $332 \mathrm{H}$.

'Abd al-Rahmān b. Yahyà el sordo (del que no se conoce acuñación).

Muhammad b. Ahmad b. Hudayr del 332 al $334 \mathrm{H}$.

'Abd Allāh b. Muhammad al-Jarrūbĩ en 335-336 H.

'Abd al-Raḥman b. Yahyyà el sordo (sin acuñación).

Muhammad ( $\dot{c}$ b. Ahmad b. Hudayr ?) del 336 al 346 H.

Ahmad en los años 346 al $350^{\circ} \mathrm{H}$.

(22) No debe olvidarse que el tomo V del Muqtabis, se interrumpe en el año $330 \mathrm{H}$., y la mención que se hace sobre la ceca y sus prefectos, recoge información general, pero es presumible que exista más, sobre este aspecto, en el resto de su obra.

(23) Conocemos alguna situación semejante en el reinado de su sucesor al-Hakam II. Cfr. Canto, A.: "Las monedas del período 361-362 H., de la ceca de Madinat al-Zahrä’”, Boletín Mus. Arq. Nacional 3, n. 2 (1985), págs. 205-210. 